\title{
14
}

\section{Petri net modelling for dynamic process planning}

\author{
Dimitris Kiritsis \\ Swiss Federal Institute of Technology - Lausanne \\ DGM-IMECO-LCAO, CH-1015 Lausanne, Switzerland \\ Dimitris.Kiritsis@imeco.dgm.epfl.ch
}

\begin{abstract}
In this paper a generic dynamic model for process planning is presented, based on Petri net technology. The proposed Petri net model 1) represents manufacturing knowledge of the type of precedence relations constraints 2 ) represents dynamically the process planning procedure itself, and 3) encapsulates all possible process planning solutions (process plans). Furthermore, process planning simulation can be performed directly on the Petri net, and finally, reachability analysis will give the complete set of solutions (process plans).
\end{abstract}

\section{Keywords}

Process planning, Petri nets, simulation, reachability analysis, CAPP, manufacturing constraints

\section{INTRODUCTION}

Knowledge based approach predominated the research work on CAPP during the last years. A recent review showed that knowledge representation and dynamic modelling techniques are key topics of intelligent and integrated process planning systems (Kiritsis, 1993). Dynamic process planning is indispensable for its integration with production planning, the immediate higher level of the global manufacturing planning hierarchy (Iwata and Fukuda, 1992).

Petri nets have been extensively used for modelling Discrete Event systems and FMSs. For a review of Petri net applications in manufacturing and a complete list of related references see the paper of Cecil, et.al (1992). Tönshoff et.al. (1987), Srihari and Emerson (1990), and Kruth and Detand (1992) presented Petri net models integrating job-shop scheduling and process planning. In these works, process planning is seen as part of a more global production planning 
system and more attention is paid to job-shop scheduling and production planning modelling by using Petri nets

In the present paper our attention is concentrated into the process planning problem and a new structure of a Petri net model for dynamic process planning is proposed. This model is generic in the sense that its construction is based on a set of standard generic rules and its graphic representation is similar for any part to be processed. With the proposed method two tools are used for model analysis and solutions finding: 1) the simulation tool can show visually, on the net graph, non-desirable conflict situations. Simulation on Petri nets is performed by firing it from its initial marking and observing tokens traveling through the net. 2) The reachability analysis tool gives all possible solutions (process plans) dynamically included in the Petri net.

\section{THE PROCESS PLANNING PROBLEM}

In part machining, process planning is the act of preparing detailed machining operation instructions to transform an engineering design to a final functional workpiece. The detailed plan contains the route, actions, machining parameters, machines and tools required for production.

The main input to the process planner could be an engineering drawing or a CAD model providing geometrical and technological information about the designed mechanical part. In an engineering drawing design entities are geometrical entities with technological attributes. In feature-based design systems, design entities are the so called manufacturing features. In both systems only finished part information is provided. The definition of all intermediate phases and states of any design entity is a task of the process planner.

The result of one machining operations is an intermediate or the final state of a design entity (geometrical surface or manufacturing feature) while the result of the process plan is the final part.

We accept that for each design entity (surface or manufacturing feature) one or more candidate operations may exist.

Whatever the design approach, the process planner's work is to choose or determine the best machining operations or processes and their sequence, able to realize the designed part, respecting the desired quality at the best cost.

A more or less complete list of process planning functions includes:

- selection of machining operations

- selection of tools

- selection of machine tools

- grouping of operations

- selection of fixturing systems

- sequence of machining operations

- determination of machining data

- generation of tool paths and NC programs

- calculation of machining times and costs

- document generation (process plan sheets) 
In this paper, the process planning problem refers to that of ordering the execution of a number of machining operations given 1) the machining data and associated actions for each machining operation and 2) a set of constraints.

Machining operations that can be executed in a machining center could be classified as following:

1. preparation

2. roughing

3. semi-finishing

4. finishing

5. post-finishing

From the global set of machining data and assuming that our machine tool reference is a modern machining center, the most influencing the planning aspects of the problem are:

1. cutting tool data and tool change actions

2. part positioning data and machining face change actions (table rotations)

3. the cutting operation itself.

The tool changes and table rotations are time consuming actions and influence the quality of the machined part.

The given constraints are:

1. precedence relations (PR) among design entities (DE), consequently, among machining operations (MO)

2. each machining operation must be processed only once.

The above information could come from many different sources: human experts (interactive input), data bases (CAPP systems of variant type), expert or knowledge based systems, and it can be summarized in machining tables like Table 1 where lines refer to design entities and contain candidate machining operations for each machining category (columns):

Table 1 Table associating design entities to machining operations

\begin{tabular}{|c|c|c|c|c|c|}
\hline & $\begin{array}{l}\text { PREPA- } \\
\text { RATION }\end{array}$ & ROUGHG & 1/2-FINISH & FINISH & $\begin{array}{l}\text { POST- } \\
\text { FINISH }\end{array}$ \\
\hline$\overline{\text { DE_1 }}$ & & $\begin{array}{l}\text { MO_1 } \\
\text { MO_2 }\end{array}$ & $\overline{\text { MO_1 }}$ & $\begin{array}{l}\text { MO_1 } \\
\text { MO_2 }\end{array}$ & \\
\hline DE_2 & MO_1 & $\begin{array}{l}\text { MO_1 } \\
\text { MO_2 }\end{array}$ & & MO_1 & MO_1 \\
\hline ........ & & & & & \\
\hline DE_n & & & & $\begin{array}{l}\text { MO_1 } \\
\text { MO_2 }\end{array}$ & \\
\hline
\end{tabular}


A worthwhile note at this point is that in such a table some operations are already planned partially: within a row, left-column operations must be executed before right-column operations.

Generally, precedence constraints can be summarized in precedence graphs like this:

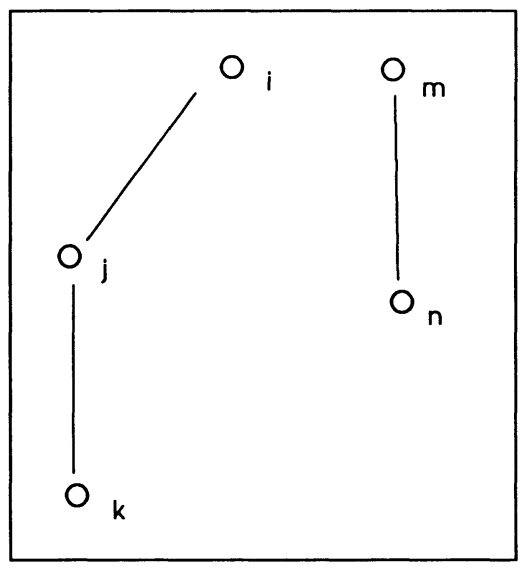

Figure 1 Precedence graph.

\section{PETRI NET MODELLING}

A Petri net consists of places and transitions which are linked to each other by directed arcs, with some arcs directed from places to transitions (input arcs), and some arcs directed from transitions to places (output arcs). A Petri net can be described as a bipartite directed graph whose nodes are a set of places and a set of transitions.

Places represent passive system components which store "tokens" and take particular states. Graphically, places are represented by circles.

Transitions represent the active system components which may produce, transport or change "tokens". For each transition there is a set of input places and a set of output places Graphically, transitions are represented by rectangles.

Arcs connect places with transitions and represent the relations between them. The arc's direction indicates the flow of information (token flow) through the net.

A transition is enabled if there is at least one token in each of its input places. When enabled, a transition removes a token from each input place and adds a token to each output place.

Tokens represent and carry information.

Marking of a Petri net is the position of tokens in the net at any instant in time. A given marking of a Petri net defines which transitions are fireable. The firing of a transition moves the net to a new marking. 
A marking $\mu^{\prime}$ is said to be reachable from a marking $\mu$ if there is a sequence of intermediate markings (and transitions) leading from $\mu$ to $\mu^{\prime}$. The set of all reachable markings from $\mu$ is called reachability set and can be represented by a reachability graph.

In this paper, we assume that:

1. only one transition can be "fired" at a time, and

2. the number of tokens in every place does not exceed one (safe Petri net).

To construct the Petri net model of a process planning problem we first analyze it and all necessary machining operations and associated resources are determined. All possible precedence relationships among machining operations are recognized and established. This is very important because it confines the number of possible solutions. For the same reason groups of operations to be executed under the same conditions are established if possible.

The following rules are applied for the proposed Petri net modelling method for process planning:

1. Each machining operation $\left(\mathrm{O}_{\mathfrak{i}}\right)$ or a well established group of them is represented by a transition, $\left(\mathrm{T}_{\mathbf{i}}\right)$.

2. Alternative (candidate) machining operations $\left(\mathrm{O}_{\mathrm{ij}}\right)$ for a design entity, if any, are represented by transitions $\left(\mathrm{T}_{\mathrm{ij}}\right)$ using the same input and output places.

3. There is a common input-output (dynamic) place (ControlPlace) with an initial token (initial marking) for all transitions representing a)the evolution of the state of the processed part (its state after every fired transition/machining operation), and b) the evolution of the state of the machine tool (tool and table position).

4. For each transition $T_{i}$ create one output place with no successor transition. This end-place $\left(\mathrm{EP}_{\mathrm{i}}\right)$, after receiving one token from the corresponding transition after its firing, indicates that this transition has already been fired and cannot be fired again.

5. For each successor transition $T_{k}$ of a transition $T_{i}$ create one output place $\left(C_{i k}\right)$ of $T_{i}$ which is an input place for the corresponding successor transition $T_{k}$. This kind of place represents the type of knowledge given by a precedence relation constraint.

6. All arcs are weighted by 1 .

A Petri net model constructed according to the above rules:

- represents accurately and dynamically the process planning procedure for a given mechanical part

- provides a graphic tool for knowledge representation of the type of precedence relations constraints, represented by the relation: transition-output place-successor transition

- provides a powerful simulation tool for process planning, simulating both machining operation sequence and state of part and machine rool

- gives all possible solutions/process plans by simulation tracing or reachability analysis

Figure 2 shows graphically the constructing elements and the basic generic structure of the proposed Petri net model for process planning. 


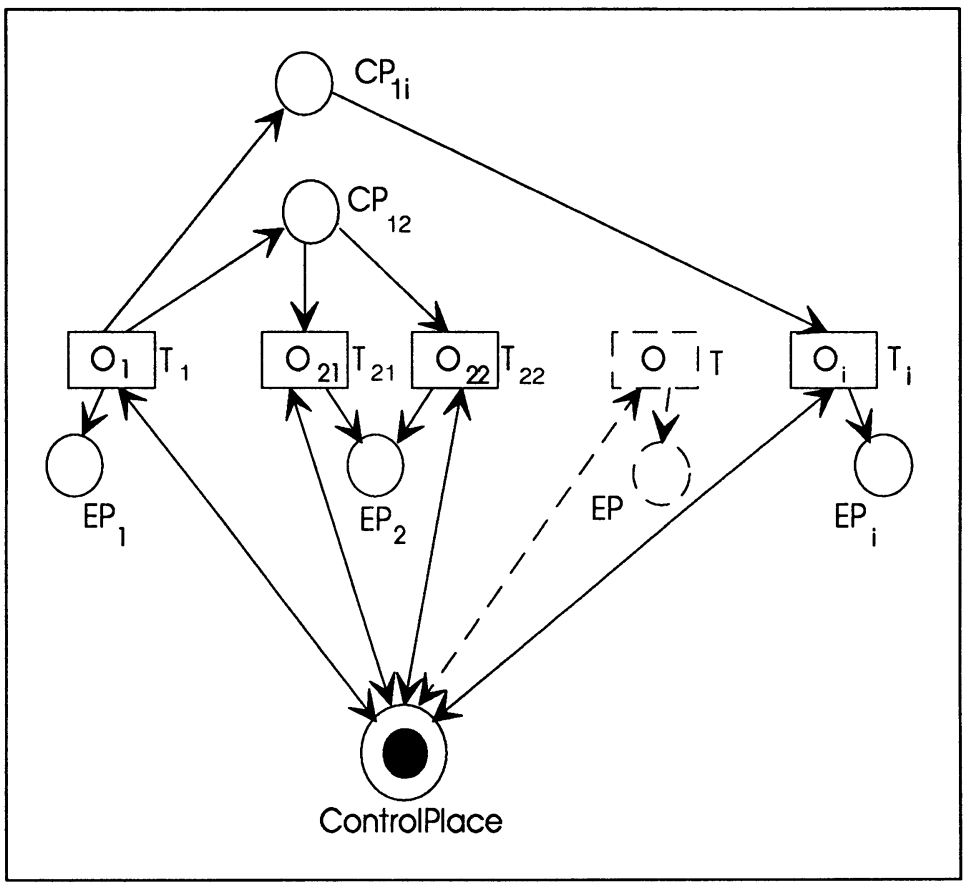

Figure 2 Principles of Petri net modelling for process planning.

\section{AN EXAMPLE}

The following example shows the principles of the proposed Petri net modelling method for process planning.

Consider the part of Figure 3. It is an "academic" prismatic workpiece consisting of five Design Entities (surfaces) to be machined in the same set-up, on the same machine tool (machining center).

\subsection{Part Analysis}

For each surface of this part, defined here as design entities (DE), the machining operations summarized in the machining table Table 2 are required. The DE 2 and 4 should be associated, i.e. machined with the same tool at the same time. 


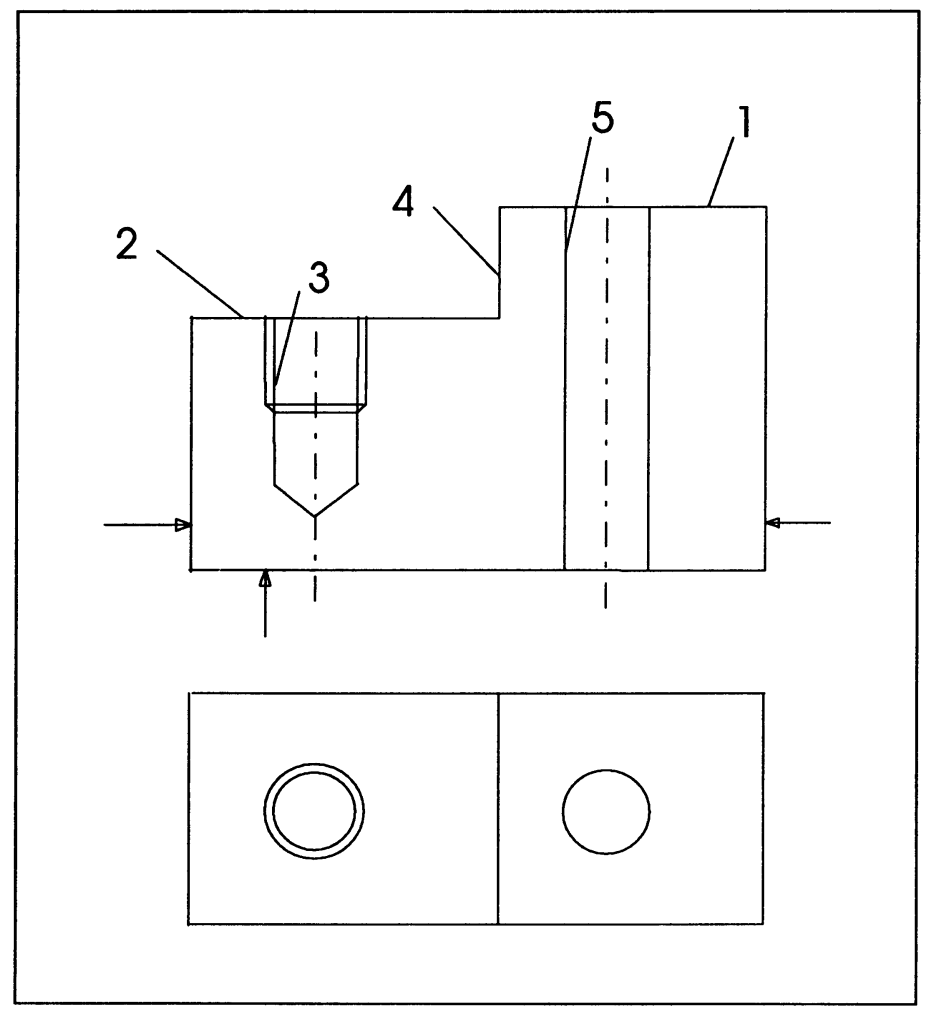

Figure 3 Example part.

Table 2 Machining table of the example of Figure 3

\begin{tabular}{|c||c|c|c|c|c|}
\hline & $\begin{array}{c}\text { PREPA- } \\
\text { RATION }\end{array}$ & ROUGH & 1/2-FINISH & FINISH & $\begin{array}{c}\text { POST- } \\
\text { FINISH }\end{array}$ \\
\hline \hline DE_1 & & 1R1 & & $1 \mathrm{~F} 1$ & \\
& & $1 \mathrm{R} 2$ & & 1F2 & \\
\hline DE_2 & & $24 \mathrm{R}$ & & $24 \mathrm{~F}$ & \\
DE 4 & & 3D & & 3T & \\
\hline DE_3 & 3C & 5D & & & \\
\hline DE_5 & 5C & 5D & & & \\
\hline
\end{tabular}

where $\mathrm{C}$ stands for Centering, $\mathrm{R}$ for Roughing (milling), $\mathrm{F}$ for Finishing (milling), D for Drilling, and $\mathrm{T}$ for Tapping. 
Candidate machining operations are defined according to the recognized possibilities to realize a DE in terms of resources (tools, machine-tools, etc.): for this example we assume 1R1 and $1 \mathrm{R} 2$ as candidate operations for roughing $\mathrm{DE} \_1$, and, $1 \mathrm{~F} 1$ and $1 \mathrm{~F} 2$ as candidate operations for finishing DE_1.

The precedence relation constraints that should be respected are:

- operation $1 F$ must be executed after operation $1 R$ and after operation $5 D$

- operation $24 R$ must be executed after operation $1 R$

- operation $24 F$ must be executed after operation $24 R$ and after operation $3 T$

- operation $3 C$ must be executed after operation $24 R$

- operation $3 D$ must be executed after operation $3 C$

- operation $3 T$ must be executed after operation $3 D$

- operation $5 C$ must be executed after operation $1 R$

- operation $5 D$ must be executed after operation $5 C$

which are summarized into the following precedence graph:

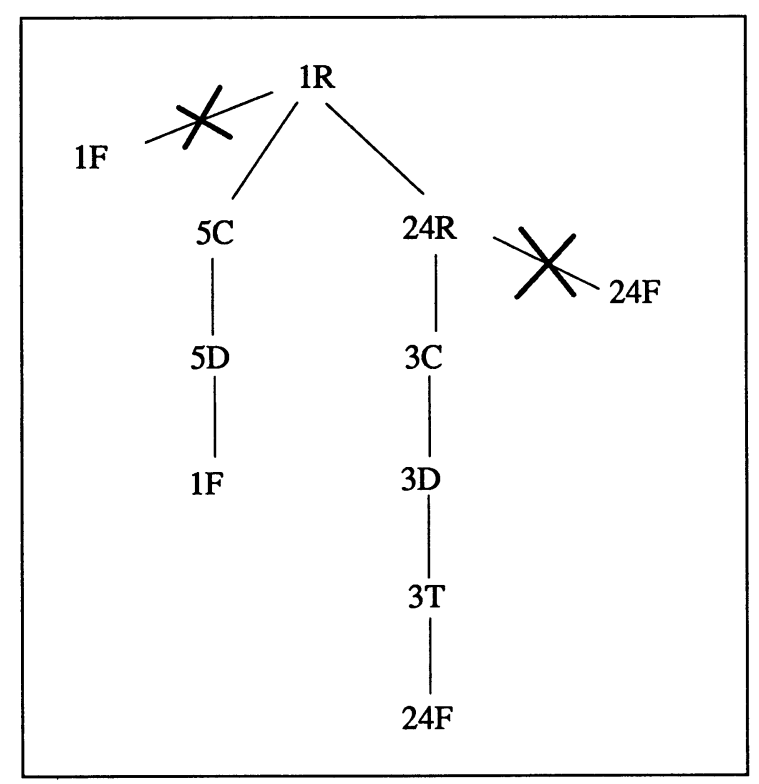

Figure 4 Precedence graph of the example of Figure 3.

By inspection of the precedence graph, we eliminate superfluous precedence constraints if any. In the above graph, for example, we can eliminate the direct precedences $1 \mathrm{R}-1 \mathrm{~F}$ and $24 \mathrm{R}-24 \mathrm{~F}$ 
because they are covered indirectly by the precedences $1 \mathrm{R}-5 \mathrm{C}-5 \mathrm{D}-1 \mathrm{~F}$ AND 24R-3C-3D-3T$24 \mathrm{~F}$ respectively. The elimination of superfluous precedence constraints is useful since it simplifies the corresponding Petri net without changing the final result.

\subsection{Petri Net Modelling}

The corresponding Petri net model is shown in Figure 5. The initial marking of this Petri net consists of one token in the common place "ControlPlace". We can simulate process planning by firing this Petri net. A process plan can be obtained after a complete simulation cycle by tracing the information carried by the token of the ControlPlace during its traveling through all transitions. Of course simulation is not an efficient method to find all possible process plans. Instead, it is a very powerful tool to detect and correct "solvable" conflict situations, if any, by adding extra constraints when this seems to be possible.

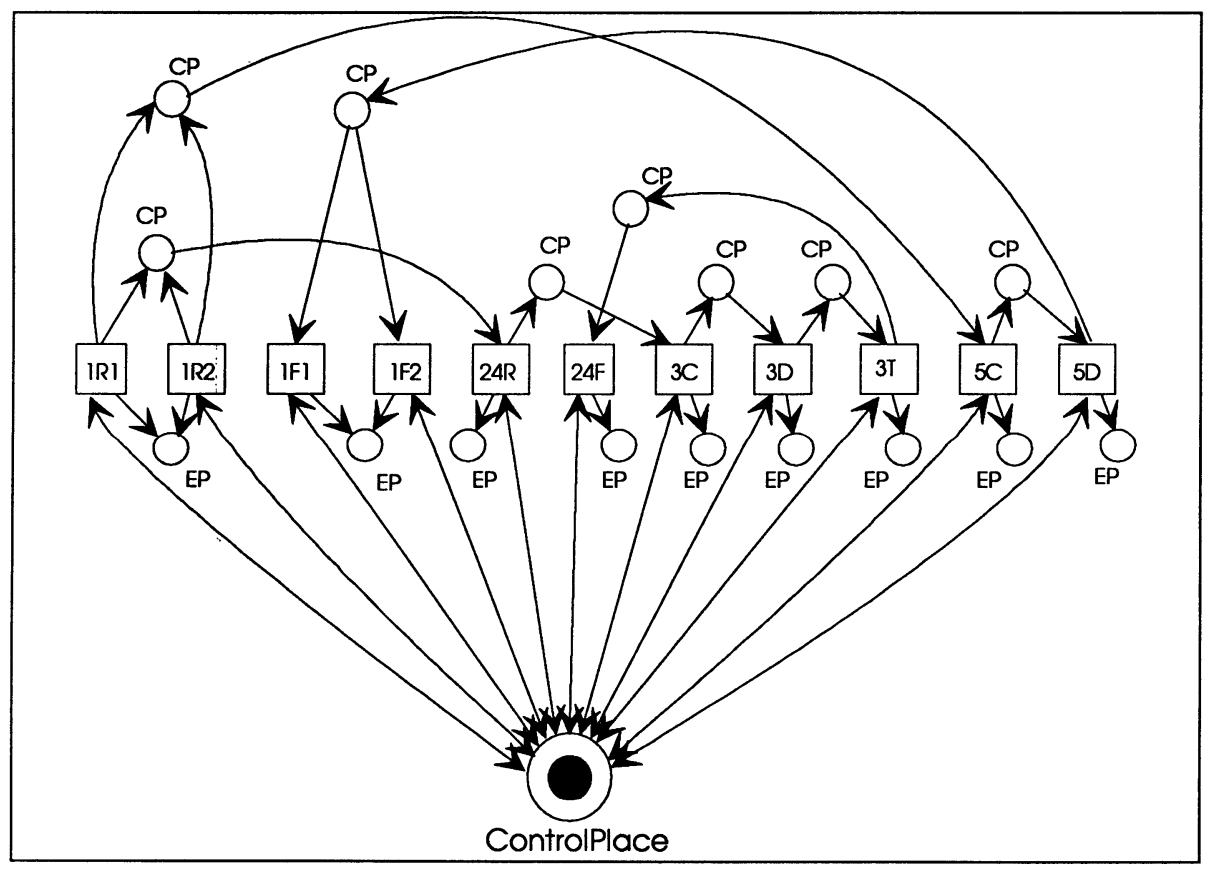

Figure 5 Petri net model of the example of Figure 3.

\subsection{Reachability Analysis}

After defining a Petri net model we can perform reachability analysis (Peterson, 1981) and calculate all alternative solutions. It is not possible to do reachability analysis manually, 
especially if the Petri net is big. We need to use software tools that perform reachability analysis automatically. Such a tool is the PROD system from the Helsinki University of Technology (Grönberg et.al., 1993). But since, with PROD, it is not possible to restrict the number of tokens of a place to one, we have to construct the equivalent of the Petri net of Figure 5 which respects PROD assumptions. This new Petri net is shown in Figure 6 and it is totally equivalent with the original Petri net of Figure 5 from the modelling and simulation point of view. Its difference with the original net is that the common place "ControlPlace" is replaced by more input places, one for each main transition. Transition corresponding to alternative candidate operations have one common input place. Input places are connected with their corresponding transitions by uni-directed arcs from the place to transition. By this method we guarantee that each transition will be fired only once. In the net of Figure 5 where the "ControlPlace" is connected with all transitions by bi-directed arcs, this is guaranteed by the assumption that the number of tokens at any place is restricted to one. The corresponding reachability graph of the Petri net of Figure 6 is shown in Figure 7. All data for creating this reachability graph were calculated automatically by using PROD.

The reachability graph represents also all possible transition sequences (arcs of the reachability graph), 84 totally, in other words all possible process plans. A list of a part of them is given in Figure 8 . This list is automatically created by applying a depth-first search algorithm (Baase, 1978) to the reachability graph of Figure 7.

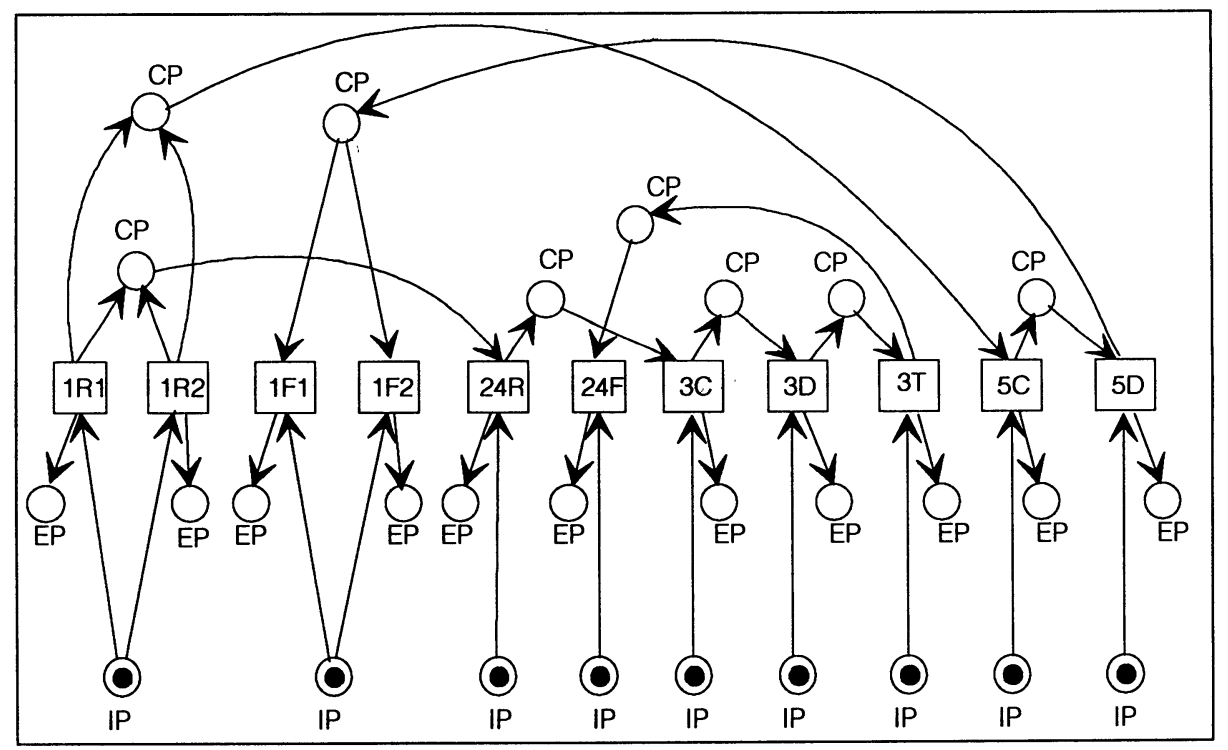

Figure 6 Modified Petri net model. 


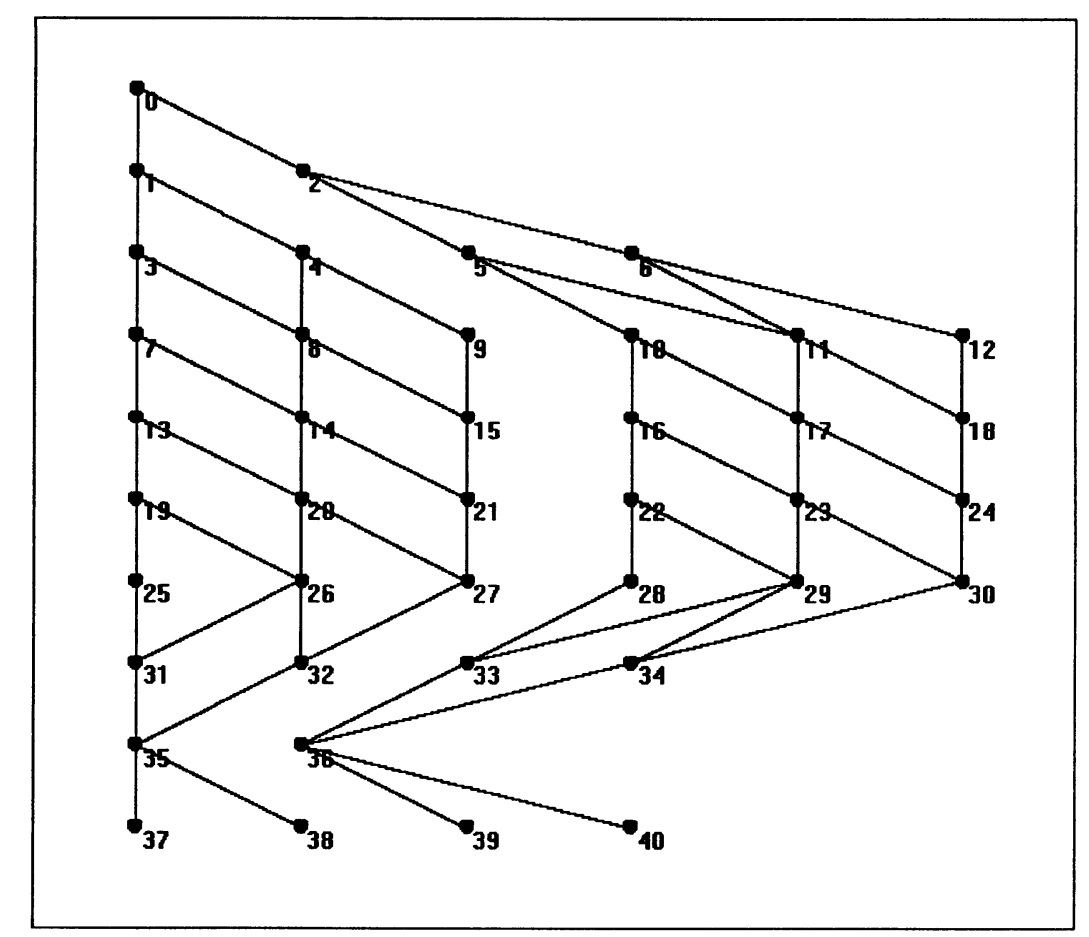

Figure 7 Reachability graph of the Petri net model of Figure 6.

\begin{tabular}{|c|}
\hline 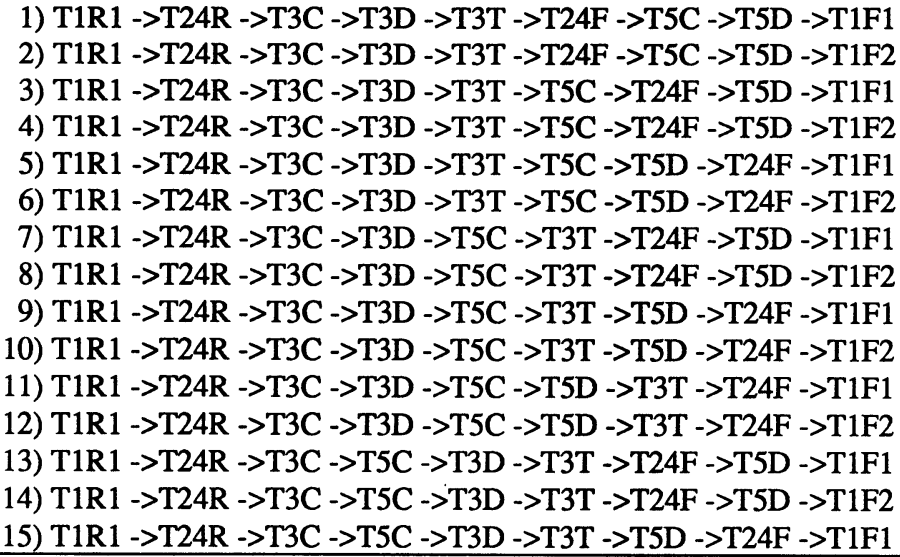 \\
\hline
\end{tabular}

Figure 8 Process plans as transition sequences (15 out of 84). 


\subsection{Finding An Optimum Solution}

It is possible that not all of the given by a reachability graph solutions are interesting. Especially in the case we have a lot (thousands) of possible solutions the finding of an optimum solution becomes combinatorial and there is a need of an optimization method using one or more heuristic criteria based on rules concerning machining actions executed in the corresponding transitions. This issue will be discussed in another paper.

\section{CONCLUSIONS}

The basic ideas of a graphic approach to process planning by using Petri nets have been discussed in this paper. A generic Petri net dynamic model of process planning has been presented and applied to an example part. The use of the proposed model is twofold. Firstly, it is a simulation tool used to detect possible problems during process planning. Secondly, reachability analysis of the Petri net gives all possible process plans for a given part under a set of given constraints. Consequently, an optimum process plan can be found by applying an optimization method to the result of the reachability analysis based on one or more heuristic criteria.

Future work will include the application of search algorithms directly to the proposed model and the introduction of time to its elements (Lee and DiCesare, 1992, Shen, et.al., 1992) The combination of Petri nets with multi-agent techniques seems to be also a promising domain of research in this direction.

Another interesting topic should be the investigation of the use of the proposed Petri net model as an element of an integrated higher level Petri net model of a production planning system (Tönshoff, et.al., 1987, 1989). This should permit the search of process planning solutions in real time according to the availability of resources at a given time (Just In Time process planning).

\section{ACKNOWLEDGMENTS}

The author would like to thank Abdelkader Belhi for his help in the example, Norbert Ebel for proposing the depth-first search algorithm, and Kimmo Varpaaniemi for his help to understand and work with PROD.

\section{REFERENCES}

Baase, S. (1978) Computer Algorithms: Introduction to Design and Analysis, Addison-Wesley Publishing Company Inc.

Cecil, J.A., Srihari, K. and Emerson, C.R. (1992) A review of Petri Net Applications in Process Planning, The International Journal of Advanced Manufacturing Technology, 7, 168-177. 
Grönberg, P., Tiusanen, M. and Varpaaniemi, K. (1993) PROD - A Pr/T-Net Reachability Analysis Tool, Technical Report, Series B, No. 11, Helsinki University of Technology, Digital Systems Laboratory.

Iwata, K. and Fukuda, Y. (1989) A New Proposal of Dynamic Process Planning in Machine Shop, CIRP International Workshop on Computer Aided Process Planning, 73-83, IFW, Hannover University.

Kiritsis, D. (1995) A Review of Knowledge-Based Expert Systems for Process Planning, The International Journal of Advanced Manufacturing Technology, accepted for publication.

Kruth, J.P. and Detand, J. (1992) A CAPP System for Nonlinear Process Plans, Annals of the CIRP, 41/1, 489-492.

Lee, D.Y. and DiCesare, F. (1992) FMS Scheduling Using Petri Nets and Heuristic Search, Proceedings of the 1992 IEEE International Conference on Robotics and Automation, 1057-1062.

Peterson, J.L. (1981) Petri Net Theory and the Modeling of Systems, Prentice-Hall Inc, Englewood Cliffs, NJ.

Shen, L., Chen, Q., Luh, J.Y.S., Chen, C. and Zhang, Z. (1992) Truncation of Petri Net Models of Scheduling Problems for Optimum Solutions, Proceedings of the JAPAN/USA Symposium on Flexible Automation, ASME, 2, 1681-1688.

Srihari, K. and Emerson, C.R. (1990) Petri Nets in Dynamic Process Planning, Computers Industrial Engineering, 19, 447-451.

Tönshoff, H.K, Beckendorff, U. and Anders, N. (1989) FLEXPLAN-A Concept for Intelligent Process Planning and Scheduling, CIRP International Workshop on Computer Aided Process Planning, 87-106, IFW, Hannover University.

Tönshoff, H.K, Beckendorff, U. and Schaelle, M. (1987) Some Approaches to Represent the Interpedence of Process Planning and Process Control,_Proceedings of the CIRP Seminars, 19th CIRP Seminar on Manufacturing Systems, Computer Aided Process Planning, Pennsylvania State University, also in Manufacturing Systems, 18/2, 93-113.

\section{BIOGRAPHY}

Dimitris Kiritsis was born in Greece in 1957. He received a diploma (1980) and a $\mathrm{PhD}$ (1987) in mechanical engineering from the University of Patras, Greece. His primary research interests are in computer-aided manufacturing, computer-aided process planning and high precision interpolation methods for CNC. He is a senior researcher at the CAD/CAM Laboratory of the Swiss Federal Institute of Technology at Lausanne, Switzerland. 